\title{
Joint DOA and DOD Estimation Based on Tensor Subspace with Partially Calibrated Bistatic MIMO Radar
}

\author{
Junxiang Wang $\mathbb{D}^{1,2}$ Ping Huang, ${ }^{1}$ and Dingjie $\mathrm{Xu}^{2}$ \\ ${ }^{1}$ College of Automation, Harbin Engineering University, Harbin, Heilongjiang 150001, China \\ ${ }^{2}$ College of Electrical Engineering and Automation, Harbin Institute of Technology University, Harbin 150001, China
}

Correspondence should be addressed to Junxiang Wang; wangjunxiang@hrbeu.edu.cn

Received 2 August 2017; Accepted 18 December 2017; Published 22 April 2018

Academic Editor: Piotr Samczynski

Copyright ( 2018 Junxiang Wang et al. This is an open access article distributed under the Creative Commons Attribution License, which permits unrestricted use, distribution, and reproduction in any medium, provided the original work is properly cited.

\begin{abstract}
A joint direction-of-departure (DOD) and direction-of-arrival (DOA) estimation algorithm based on tensor subspace approach for partially calibrated bistatic multiple-input multiple-output (MIMO) radar is proposed. By exploiting the multidimensional structure of the received data, a third-order measurement tensor is constructed. Consequently, the tensor-based signal subspace is achieved using the higher-order singular value decomposition (HOSVD). To achieve accurate DOA estimation with partially calibrated array, a closed-form solution is provided to estimate the gain-phase uncertainties of the transmit and receive arrays by modeling the imperfections of the arrays. Simulation results demonstrate the effectiveness of the proposed calibration algorithm.
\end{abstract}

\section{Introduction}

Array signal processing, which includes parameter estimation $[1,2]$ and beamforming $[3,4]$ has been widely investigated in past decades [5] which has found various applications in radar, sonar, and satellite navigation system. In recent years, multiple-input multiple-output (MIMO) radar $[6,7]$ has drawn increasing attention due to its significant advantages, such as higher angular resolution and more degrees-of-freedom (DOFs). MIMO radar using multiple antennas to simultaneously emit orthogonal waveforms and multiple antennas to receive the reflected signals has recently drawn increasing attention owing to its superior performance over to the conventional phased array. Direction-ofdeparture (DOD) and direction-of-arrival (DOA) estimation is an important objective of bistatic MIMO radar system. Various algorithms for DOD and DOA estimation have been proposed in literatures [8-12]. In [8], the rotational invariance property of the MIMO radar is exploited which enables the DOD and DOA estimation with bistatic MIMO radar. However, this method determines the DODs and DOAs using two independent estimation of signal parameters via rotational invariance technique (ESPRIT) and requires an extra procedure to achieve the paired DOD and DOA estimation. To avoid the pair matching procedure, the relationship between the two independent ESPRIT is exploited in [9] to estimate the DODs and DOAs. In [10], a reduceddimension MUltiple SIgnal Classification (MUSIC) algorithm is proposed which only requires one-dimensional peak search. This method achieves a similar performance compared with the two-dimensional MUSIC algorithm and has a lower computational complexity. Based on the double polynomial root-finding procedure, a polynomial root-finding technique for joint DOD and DOA estimation is proposed in [11]. This algorithm allows an efficient estimation with automatic pairing. In [12], the property of the Kronecker product is utilized to formulate a cost function in the quadratic form. Then the DODs can be estimated from the constraint function. The DOAs can be achieved from the eigenvector which is related to the corresponding DODs.

However, the algorithms mentioned above are based on the assumption that arrays are well calibrated which is not true in practice. Therefore, the above algorithms will suffer from performance degradation or even fail to achieve 
accurate DOD and DOA estimation in many scenarios. A number of algorithms have been proposed to deal with the array calibration problem [13-17]. An iterative algorithm based on the MUSIC technique is proposed in [13], which can simultaneously achieve the angle and gain-phase uncertainties estimation. Due to the iterative procedure, this algorithm has a high computational complexity. In addition, this method is based on the assumption that the gain-phase uncertainties are small, otherwise it will suffer from suboptimal convergence. The algorithms proposed in $[15,16]$ do not require iteration and exploit the ESPRIT-like technique to achieve accurate angle estimations. To achieve higher DOFs, the method proposed in [17] utilizes the property of the quasistationary signals to solve the underdetermined DOA estimation problem with partly calibrated arrays. Moreover, a kind of calibration methods based on the sparse reconstruction is discussed in $[18,19]$.

However, the multiple-dimensional property of the received signal is not exploited in the abovementioned algorithms. The inherently multiple-dimensional structure of the received signal can be utilized by expressing the received signal as a third-order tensor [20]. Thus, the higher-order singular value decomposition (HOSVD) can be used to achieve a tensor-based signal subspace which improves the estimation performance especially with low signal to noise ratio (SNR).

In this paper, we exploit a calibration algorithm based on tensor approach for partially calibrated bistatic MIMO radar. We first exploit the multidimensional structure of the received data, and then, the tensor-based signal subspace is calculated using HOSVD. By formulating the gain-phase imperfections in the generalized manifold matrix, a closed-form solution is achieved to estimate the gain-phase uncertainties of the transmit and receive arrays. With the estimated gain-phase uncertainties, the arrays can be calibrated and the DODs and DOAs of the sources can be estimated accurately. Simulation results demonstrate the effectiveness of the proposed joint DOD and DOA estimation algorithm for partially calibrated bistatic MIMO radar.

The rest of this paper is organized as follows. In Section 2, we briefly introduce the tensor algebra basics and the tensor-based signal model of the bistatic MIMO radar. In Section 3, we first describe the partially calibrated MIMO radar model. Then, the proposed method is introduced. Numerical simulation results are presented in Section 4. The conclusions are made in Section 5.

Notations used in this paper are as follows: lowercase (uppercase) bold characters are used to denote vectors (matrices). $[\mathcal{A}]_{i, j, k}$ stands for the $(i, j, k)$ th entry of $\mathcal{A}$. $\mathbf{I}_{N}$ denotes the $N \times N$ identity matrix. $(\cdot)^{T}$ and $(\cdot)^{H}$ denote the transpose and Hermitian operations of a matrix or vector, respectively. $\operatorname{vec}(\cdot)$ stands for the vectorization operator. $\operatorname{diag}(\mathbf{x})$ denotes a diagonal matrix with the elements of $\mathbf{x}$ constituting the diagonal entries. $\otimes$ denotes the Kronecker product, $\circ$ denotes the Hadamard product, and $\odot$ denotes the Khatri-Rao product. In addition, $\|\cdot\|_{2}$ denotes the $\ell_{2}$-norm, whereas $\mathbb{C}^{M \times N}$ and $\mathbb{C}^{M}$ express an $M \times N$ complex matrix or $M \times 1$ vector set.

\section{Tensor Algebra Basic and Signal Model}

In this section, we first introduce tensor operations used in this paper and provide the bistatic MIMO radar signal model.

2.1. Tensor Algebra Basic. In this subsection, the tensor algebra basic is introduced for readers' convenience [20, 21].

Definition 1 ( $n$-mode unfolding). Denote a $N$-order tensor as $\mathcal{A} \in I_{1} \times I_{2} \times \cdots \times I_{n}$, the corresponding $n$-mode unfolding is to convert $\mathcal{A}$ into a matrix which is expressed as $[\mathcal{A}]_{(n)}$ $\in \mathbb{C}^{I_{n} \times I_{1} I_{2} \cdots I_{n-1} I_{n+1} \cdots I_{N}}$. The $\left(i_{1}, i_{2}, \ldots, i_{N}\right)$ th entry of the tensor $\mathcal{A}$ maps to the $\left(i_{n}, j\right)$ entry of $[\mathcal{A}]_{(n)}$, where

$$
j=1+\sum_{k=1, k \neq 1}^{N}\left(i_{k}-1\right) J_{k}, \quad \text { with } J_{k}=\prod_{m=1, m \neq n} i_{m} .
$$

Definition 2 ( $n$-mode tensor product). The $n$-mode matrix product of the tensor $\mathcal{A} \in I_{1} \times I_{2} \times \cdots \times I_{n}$ with a matrix $\mathbf{U}_{n} \in$ $\mathbb{C}^{J \times I_{n}}$ is denoted by $\mathcal{Y}=\mathcal{A} \times{ }_{n} \mathbf{U}_{n}$ with size $I_{1} \times I_{2} \times \cdots \times I_{n-1}$ $\times J \times I_{n+1} \times \cdots \times I_{N}$ and

$$
\begin{array}{r}
{[\mathscr{Y}]_{i_{1}, \ldots, i_{n-1}, j, i_{n+1}, \ldots, i_{N}}=\sum_{i_{n}=1}^{I_{n}} \mathscr{X}_{i_{1}, i_{2}, \ldots, i_{N}} \mathbf{U}_{j, i_{n}},} \\
\mathscr{Y}=\mathscr{X} \times{ }_{n} \mathbf{U} \Leftrightarrow[\mathcal{Y}]_{(n)}=\mathbf{U}[\mathscr{X}]_{(n)} .
\end{array}
$$

Moreover, the properties of the mode tensor product are provided below:

$$
\begin{aligned}
& \mathscr{X} \times{ }_{m} \mathbf{U}_{m} \times{ }_{n} \mathbf{U}_{n}=\mathscr{X} \times{ }_{n} \mathbf{U}_{n} \times{ }_{m} \mathbf{U}_{m}, \quad m \neq n, \\
& \mathscr{X} \times{ }_{m} \mathbf{U} \times{ }_{m} \mathbf{V}=\mathscr{X} \times{ }_{n}(\mathbf{U V}) .
\end{aligned}
$$

Definition 3 (HOSVD). The HOSVD of the tensor A

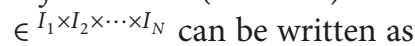

$$
\mathbf{A}=\mathbf{S} \times{ }_{1} \mathbf{U}_{1} \times{ }_{2} \mathbf{U}_{2} \times{ }_{3} \cdots \times{ }_{N} \mathbf{U}_{N},
$$

where $\mathbf{S} \in \mathbb{C}^{I_{1} \times I_{2} \times \cdots \times I_{n}}$ is the core tensor of $\mathcal{A}$ which can be calculated by

$$
\mathbf{S}=\mathbf{A} \times{ }_{1} \mathbf{U}_{1}^{H} \times{ }_{2} \mathbf{U}_{2}^{H} \times{ }_{3} \cdots \times{ }_{N} \mathbf{U}_{N}^{H} .
$$

In addition, $\mathbf{U}_{n}, n=1, \ldots, N$, is a unitary $I_{n} \times I_{n}$, which is the left singular matrix of $\mathbf{U}_{n}$.

2.2. Bistatic MIMO Radar Signal Model. Consider a bistatic MIMO radar system where the transmit and receive arrays are both uniform linear arrays (ULAs) with $M$ and $N$ colocated antennas, respectively. In addition, both the transmit and receive antennas are arranged with half-wavelength interelement space. The transmit antennas simultaneously emit orthogonal waveforms $\mathbf{S}=\left[\mathbf{s}_{1}^{T}, \ldots, \mathbf{s}_{M}^{T}\right]^{T} \in \mathbb{C}^{M \times L}$ where $L$ is the number of samples during pulse period. Assume that there are $K$ uncorrelated narrowband far-field source signals in the range bin of interest. The baseband received signal at the $q$ th pulse can be expressed as:

$$
\mathbf{X}_{q}=\mathbf{A}_{r} \boldsymbol{\Sigma}_{q} \mathbf{A}_{t}^{T} \mathbf{S}+\mathbf{Z}_{q}, \quad q=1, \ldots, Q,
$$


where $\mathbf{A}_{r}=\left[\mathbf{a}_{r}\left(\theta_{1}\right), \ldots, \mathbf{a}_{r}\left(\theta_{K}\right)\right]$ and $\mathbf{A}_{t}=\left[\mathbf{a}_{t}\left(\phi_{1}\right), \ldots, \mathbf{a}_{t}\left(\phi_{K}\right)\right]$ are the receive and transmit manifold matrix, respectively. $\mathbf{a}_{r}\left(\theta_{k}\right)=\left[1, e^{-j \pi \sin \theta_{k}}, \ldots, e^{-j \pi\left(M_{r}-1\right) \sin \theta_{k}}\right]^{T} \quad$ and $\quad \mathbf{a}_{t}\left(\phi_{k}\right)=$ $\left[1, e^{-j \pi \sin \phi_{k}}, \cdots, e^{-j \pi\left(M_{t}-1\right) \sin \phi_{k}}\right]^{T}$ are the steering vectors of the $k$ th source, respectively. $\left\{\theta_{k}, \phi_{k}\right\}_{k=1}^{K}$ is the pair of the DOA and DOD of the $k$ th source. $\Sigma_{q}=\operatorname{diag}\left(\mathbf{c}_{q}\right)$ with $\mathbf{c}_{q}=$ $\left[\gamma_{1 q}, \ldots, \gamma_{K q}\right]^{T}$ accounts for the Doppler effect and the radar reflection coefficient fading which follows the Swerling I model [22]. Finally, $Z_{q} \in \mathbb{C}^{M_{r} \times L}$ is zero mean Gaussian noise term. Under the fact that the transmitted waveforms are orthogonal to each other, that is,

$$
\left(\frac{1}{L}\right) \mathbf{s}_{i} \mathbf{s}_{j}^{H}=\left\{\begin{array}{l}
1, i=j, \\
0, i \neq j,
\end{array} \quad i, j=1, \ldots, M_{t} .\right.
$$

Then, the received signal can be processed by matched filtering using $\mathbf{S}^{H}$. Thus, the output of the matched filter can be written as

$$
\mathbf{Y}_{q}=\left(\frac{1}{L}\right) \mathbf{X}_{q} \mathbf{S}^{H}=\mathbf{A}_{r} \boldsymbol{\Sigma}_{q} \mathbf{A}_{t}^{T}+\mathbf{N}_{q}, \quad q=1, \ldots, Q,
$$

where $\mathbf{N}_{q}=(1 / L) \mathbf{Z}_{q} \mathbf{S}^{H}$ is additive zero mean Gaussian noise term with unknown variance $\sigma_{n}^{2}$ after matched filtering.

Usually, the received signal matrix can be achieved by vectorizing and stacking the $Q$ pulses received signal, that is,

$$
\mathbf{Y}=\left[\operatorname{vec}\left(\mathbf{Y}_{1}\right), \ldots, \operatorname{vec}\left(\mathbf{Y}_{Q}\right)\right]=\left(\mathbf{A}_{t} \odot \mathbf{A}_{r}\right) \mathbf{C}+\mathbf{N},
$$

where $\mathbf{C}=\left[\mathbf{c}_{1}, \ldots, \mathbf{c}_{Q}\right]$ and $\mathbf{N}=\left[\operatorname{vec}\left(\mathbf{N}_{1}\right), \ldots, \operatorname{vec}\left(\mathbf{N}_{Q}\right)\right]$. However, this expression ignores the multidimensional structure inherent of the received signals. In order to exploit the multidimensional structure of the received signal, we collect $Q$ pulses and gather $\mathbf{Y}_{q}, q=1, \ldots, Q$, as a third-order tensor given by

$$
\mathcal{Y}=\mathscr{I}_{K} \times{ }_{1} \mathbf{A}_{r} \times{ }_{2} \mathbf{A}_{t} \times{ }_{3} \mathbf{C}^{T}+\mathcal{N},
$$

where $\mathbf{I}_{K}$ is the $K \times K \times K$ identity tensor. $\mathbf{N} \in \mathbb{C}^{M_{r} \times M_{t} \times Q}$ is the noise tensor. According to Definition 1, the relationship between (8) and (10) can be expressed as $[Y]_{(3)}^{T}=Y$.

\section{Proposed Method}

In this section, the partially calibrated MIMO radar signal model is first provided. Then, a robust DOA estimation algorithm based on tensor algebra is presented.

3.1. Partially Calibrated MIMO Radar Model. Consider the case where only a part of the transmit and receive antennas is well-calibrated. Without loss of generality, we assume that the first $M_{t c}$ and $M_{r c}$ antennas of the transmit and receive arrays are calibrated, respectively, whereas the rest of the sensors are uncalibrated with unknown gain-phase uncertainties. Moreover, we assume that the uncertainties are direction-independent. Thus, the transmit manifold matrix and the receive manifold matrix can be, respectively, expressed as

$$
\begin{aligned}
\tilde{\mathbf{A}}_{t} & =\Gamma\left(\gamma_{t}\right) \mathbf{A}_{t}, \\
\tilde{\mathbf{A}}_{r} & =\Gamma\left(\gamma_{r}\right) \mathbf{A}_{r},
\end{aligned}
$$

where $\Gamma\left(\gamma_{t}\right)=\operatorname{diag}\left(\gamma_{t}\right)$ and $\Gamma\left(\gamma_{r}\right)=\operatorname{diag}\left(\gamma_{r}\right)$ with gain-phase uncertainties $\gamma_{t}=\left[1_{M_{t c}}^{T}, \rho_{1} e^{j \varphi_{1}}, \ldots, \rho_{M_{t}-M_{t c}} e^{\left.j \varphi_{M_{t}-M_{t c}}\right]^{T}}\right.$ and $\gamma_{r}=$ $\left[1_{M_{r c}}^{T}, \alpha_{1} e^{j \beta_{1}}, \ldots, \alpha_{M_{r}-M_{r c}} e^{\left.j \beta_{M_{r}-M_{r c}}\right]^{T}}\right.$, respectively. Thus, the received signal matrix can be expressed as

$$
\tilde{\mathscr{Y}}=\mathbf{I}_{K} \times{ }_{1} \tilde{\mathbf{A}}_{r} \times{ }_{2} \tilde{\mathbf{A}}_{t} \times{ }_{3} \mathbf{C}+\mathscr{N} .
$$

For the case of partially calibrated array, the conventional DOD and DOA estimation algorithm will suffer from severe performance degradation. In order to achieve satisfactory angle estimation result, the partially calibrated problem should be concerned.

3.2. ESPRIT-Like Calibration Method. The HOSVD of the received signal (12) can be written as

$$
\tilde{y}=\delta \times{ }_{1} \mathbf{U}_{1} \times{ }_{2} \mathbf{U}_{2} \times{ }_{3} \mathbf{U}_{3},
$$

where $\mathcal{S} \in \mathbb{C}^{M_{r} \times M_{t} \times Q}, \mathbf{U}_{1} \in \mathbb{C}^{M_{r} \times M_{r}}, \mathbf{U}_{2} \in \mathbb{C}^{M_{t} \times M_{t}}$, and $\mathbf{U}_{3} \in$ $\mathbb{C}^{Q \times Q}$. Denote the signal subspace of $\mathscr{Y}$ as

$$
\mathcal{U}_{s}=\mathcal{S}_{s} \times{ }_{1} \mathbf{U}_{1 s} \times{ }_{2} \mathbf{U}_{2 s} \text {, }
$$

where $\mathcal{S}_{s}=\tilde{y} \times{ }_{1} \mathbf{U}_{1 s}^{H} \times{ }_{2} \mathbf{U}_{2 s}^{H} \times{ }_{3} \mathbf{U}_{3 s}^{H}, \mathbf{U}_{n s}$ contains the first $K$ dominant singular vectors of $\mathbf{U}_{n}$ with $n=1,2,3$. Thus, according to Definition 3, we have

$$
\boldsymbol{U}_{s}=\tilde{\mathscr{y}} \times{ }_{1}\left(\mathbf{U}_{1 s} \mathbf{U}_{1 s}^{H}\right) \times{ }_{2}\left(\mathbf{U}_{2 s} \mathbf{U}_{2 s}^{H}\right) \times{ }_{3} \mathbf{U}_{3 s}^{H} .
$$

Then, the signal subspace in matrix form can be calculated from the 3-mode unfolding of $\mathcal{U}_{s}$, that is, $\left[U_{s}\right]_{(3)}^{T}=\mathbf{E}_{s}$. Note that the signal subspace expands the same space with the manifold matrix, that is,

$$
\mathbf{E}_{s}=\left(\tilde{\mathbf{A}}_{t} \odot \tilde{\mathbf{A}}_{r}\right) \mathbf{T},
$$

where $\mathbf{T}$ is a full rank matrix.

The generalized manifold matrix can be rewritten as

$$
\tilde{\mathbf{A}}_{t} \odot \tilde{\mathbf{A}}_{r}=\Gamma\left(\gamma_{t} \otimes \gamma_{r}\right)\left(\mathbf{A}_{t} \odot \mathbf{A}_{r}\right)=\Gamma(\tilde{\gamma})\left(\mathbf{A}_{t} \odot \mathbf{A}_{r}\right),
$$

where $\tilde{\gamma}=\gamma_{t} \otimes \gamma_{r}$. Thus, by selecting the first $\left(M_{t}-1\right) M_{r}$ rows of $\mathbf{E}_{s}$, we have

$$
\begin{aligned}
& \mathbf{E}_{t 1}=\left(\tilde{\mathbf{A}}_{t 1} \odot \tilde{\mathbf{A}}_{r}\right) \mathbf{T}=\Gamma\left(\tilde{\gamma}_{t 1}\right)\left(\mathbf{A}_{t 1} \odot \mathbf{A}_{r}\right) \mathbf{T}, \\
& \mathbf{E}_{t 2}=\left(\tilde{\mathbf{A}}_{t 2} \odot \tilde{\mathbf{A}}_{r}\right) \mathbf{T}=\Gamma\left(\tilde{\gamma}_{t 2}\right)\left(\mathbf{A}_{t 2} \odot \mathbf{A}_{r}\right) \mathbf{T},
\end{aligned}
$$

where $\tilde{\mathbf{A}}_{t 1}$ and $\tilde{\mathbf{A}}_{t 2}$ are the first and last $\left(M_{t}-1\right) M_{r}$ rows of $\tilde{\mathbf{A}}_{t}$, respectively. $\tilde{\gamma}_{t 1}$ and $\tilde{\gamma}_{t 2}$ denote the first and last $\left(M_{t}-1\right) M_{r}$ entries of $\tilde{\gamma}$, respectively. Obviously, $\mathbf{A}_{t 1}$ and $\mathbf{A}_{t 2}$ has the rotational invariance property, that is, $\mathbf{A}_{t 2}=\mathbf{A}_{t 1}$ $\boldsymbol{\Phi}_{t}$ with $\boldsymbol{\Phi}_{t}=\operatorname{diag}\left(\left[e^{-j \pi \sin \phi_{1}}, \cdots, e^{-j \pi \sin \phi_{K}}\right]\right)$. Thus, we have the following expression 


$$
\Gamma\left(\tau_{t}\right) \mathbf{E}_{t 2}=\mathbf{E}_{t 1} \Psi_{t},
$$

where $\boldsymbol{\Psi}_{t}=\mathbf{T}^{-1} \boldsymbol{\Phi}_{t} \mathbf{T}$ and

$$
\begin{aligned}
\boldsymbol{\tau}_{t}= & \tilde{\boldsymbol{\gamma}}_{t 1} \circ \tilde{\boldsymbol{\gamma}}_{t 2}^{-1}=\left(\boldsymbol{\gamma}_{t 1} \circ \boldsymbol{\gamma}_{t 2}^{-1}\right) \otimes 1_{M_{r}} \\
= & {\left[1_{M_{t c}-1}^{T},\left(\rho_{1} e^{j \phi_{1}}\right)^{-1}, \rho_{1} e^{j \phi_{1}}\left(\rho_{2} e^{j \phi_{2}}\right)^{-1}, \ldots,\right.} \\
& \left.\rho_{M_{t}-M_{t c}-1} e^{j \phi_{M_{t}-M_{t c}-1}}\left(\rho_{M_{t}-M_{t c}} e^{j \phi_{M_{t}-M_{t c}}}\right)^{-1}\right]^{T} \otimes 1_{M_{r}}
\end{aligned}
$$

with $\gamma_{t 1}$ and $\gamma_{t 2}$ denoting the first and last $M_{t-1}$ entries of $\gamma_{t}$, respectively. Once the matrix $\boldsymbol{\Psi}_{t}$ is calculated, the DODs of the sources can be estimated. Thus, we construct the following optimization problem

$$
\begin{aligned}
& \min _{\boldsymbol{\Psi}_{t}, \boldsymbol{\tau}_{t}} \quad\left\|\Gamma\left(\tau_{t}\right) \mathbf{E}_{t 2}-\mathbf{E}_{t 1} \Psi_{t}\right\|_{2}^{2} \\
& \text { s.t. } \quad \mathbf{W}_{t} \tau_{t}=1_{(M t c-1) M_{r}},
\end{aligned}
$$

where $\mathbf{W}_{t}=\left[\mathbf{I}_{(M t c-1) M_{r}}, 0_{(M t c-1) M_{r} \times\left(\left(M_{t}-1\right) M_{r}-(M t c-1) M_{r}\right)}\right]$ is used to ensure that the first $M_{t c}$ sensors of the transmit array are well calibrated. $\Psi_{t}$ as

From (19), we can achieve the least square solution of

$$
\boldsymbol{\Psi}_{t}=\left(\mathbf{E}_{t 1}^{H} \mathbf{E}_{t 1}\right)^{-1} \mathbf{E}_{t 1}^{H} \Gamma\left(\tau_{t}\right) \mathbf{E}_{t 2} .
$$

By substituting (22) into (21), we have

$$
\begin{array}{ll}
\min _{\mathbf{\Psi}_{t}, \boldsymbol{\tau}_{t}} & \left\|\mathbf{P}_{t} \Gamma\left(\tau_{t}\right) \mathbf{E}_{t 2}\right\|_{2}^{2} \\
\text { s.t. } & \mathbf{W}_{t} \tau_{t}=1_{(M t c-1) M_{r}},
\end{array}
$$

where $\mathbf{P}_{t}=\mathbf{I}_{\left(M_{t}-1\right) M_{r}}-\mathbf{E}_{t 1}\left(\mathbf{E}_{t 1}^{H} E_{t 1}\right)^{-1} \mathbf{E}_{t 1}^{H}$.

Utilizing the fact that $\|\mathbf{A}\|_{F}^{2}=\operatorname{trace}\left\{\mathbf{A}^{H} \mathbf{A}\right\}$, $\operatorname{trace}(\mathbf{A B})=$ trace $(\mathbf{B A})$, and $\operatorname{trace}\{\mathbf{A} \Gamma(\mathbf{d}) \mathbf{B} \Gamma(\mathbf{d})\}=\mathbf{d}^{H}\left(\mathbf{A}^{T} \circ \mathbf{B}\right) \mathbf{d}$ for matrices $\mathbf{A}, \mathbf{B}$, and vectors $\mathbf{d}$ with proper dimensions, the objective function in (23) is further simplified as

$$
\left\|\mathbf{P}_{t} \Gamma\left(\tau_{t}\right) \mathbf{E}_{t 2}\right\|_{2}^{2}=\tau_{t}^{H}\left(\left(\mathbf{E}_{t 2} \mathbf{E}_{t 2}^{H}\right)^{T} \circ \mathbf{P}_{t}\right) \tau_{t}
$$

Thus, the optimization in (23) is expressed as

$$
\begin{aligned}
& \min _{\tau_{t}} \tau_{t}^{H}\left(\left(\mathbf{E}_{t 2} \mathbf{E}_{t 2}^{H}\right)^{T} \circ P_{t}\right) \tau_{t} \\
& \text { s.t. } \mathbf{W}_{t} \tau_{t}=1_{(M t c-1) M_{r}} .
\end{aligned}
$$

This problem can be solved through the Lagrange multiplier method and the optimal solution is expressed as:

$$
\widehat{\tau}_{t}=\mathbf{Q}_{t}^{-1} \mathbf{W}_{t}^{T}\left(\mathbf{W}_{t} \mathbf{Q}_{t}^{-1} \mathbf{W}^{T}\right)^{-1} 1_{\left(M_{t c}-1\right) M_{r}},
$$

where $\mathbf{Q}=\left(\mathbf{E}_{t 2} \mathbf{E}_{t 2}^{H}\right)^{T} \circ \mathbf{P}_{t}$. Thus, $\boldsymbol{\Psi}_{t}$ can be estimated through (22). Note that $\boldsymbol{\Psi}_{t}=T^{-1} \boldsymbol{\Phi}_{t} T$, we can use eigenvalue decomposition to achieve the estimation of $\Phi_{t}$ and the DOD estimation of the $k$ th source can be calculated as

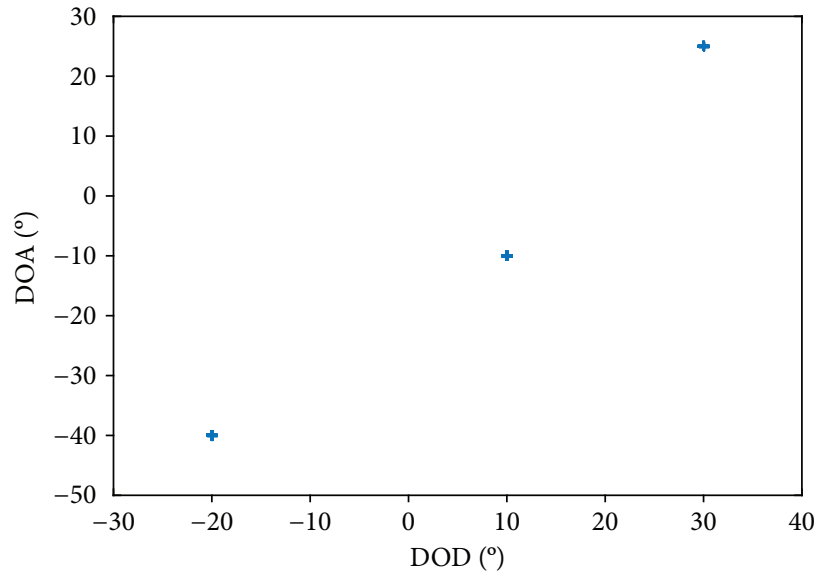

FIGURE 1: 100 realizations of the proposed method. $\mathrm{SNR}=5 \mathrm{~dB}$.

$$
\widehat{\phi}_{k}=\arcsin \left(-\frac{\operatorname{angle}\left(v_{t, k}\right)}{\pi}\right), \quad k=1, \ldots, K,
$$

where $v_{t, k}$ is the $k$ th eigenvalue of $\boldsymbol{\Phi}_{t}$.

In addition, the $m$ th element of $\gamma_{t}$ also can be estimated as

$$
\widehat{\gamma}_{t, m}=\left(\prod_{i=1}^{m} \varepsilon(i)\right)^{-1}, \quad m=N_{t c}+1, \ldots, M_{t},
$$

where $\varepsilon(i)=1 / M_{r} \sum_{l=(i-1) M_{r}+1}^{i M_{r}} \widehat{\tau}_{t, l}, \widehat{\tau}_{t, l}$ is the $l$ th elements of $\widehat{\tau}_{t}$.

We select a transformation matrix $\mathbf{J}$ which makes $\mathbf{E}_{s}^{\prime}=\mathbf{J E}_{s}=\left(\tilde{\mathbf{A}}_{r} \odot \tilde{\mathbf{A}}_{t}\right) \mathbf{T}$, then the DOAs of the sources can be estimated by using a similar manner with the estimation of DODs. Thus, the detailed procedure is omitted in this paper. Furthermore, the DODs and DOAs can be paired using the method proposed in [9].

\section{Simulation Results}

In this section, several experimental simulations are carried out to demonstrate the performance of the proposed method.

A bistatic MIMO radar with $M_{t}=6$ transmit antennas and $M_{r}=8$ receive antennas is used in the following simulations. In addition, both the transmit and receiver arrays are assumed to be partially calibrated. The corresponding gainphase uncertainties are $\gamma_{t}=\left[1,1,1,0.8 e^{j 0.6283}, 1.25 e^{-j 1.0472}\right.$, $\left.0.75 e^{j 0.7854}\right]^{T}$ and $\gamma_{r}=\left[1,1,1.3712 e^{-j 0.2675}, 1.1199 e^{j 0.0676}, 1\right.$, $\left.0.9730 e^{-j 0.2509}, 1,0.96 e^{j 0.0381}\right]^{T}$. Thus, we have $M_{t c}=3$ and $M_{r c}=2$. The number of samples during one pulse period is $L=256$, and the number of pulses is set as $Q=200$ unless otherwise specified.

In the first simulation, 100 realizations of the proposed method are provided in Figure 1. In this simulation, the SNR is set as $5 \mathrm{~dB}$. From Figure 1, it is clear that the proposed method achieves accurate DOD and DOA estimation which are paired correctly. The estimation of gain-phase uncertainties are provided in Table 1. The mean and standard deviation (STD) of the estimations are calculated from 100 independent Monte Carlo trials. 
TABLE 1: Performance of gain and phase estimation.

(a) Gain estimation results of $\gamma_{t}$

\begin{tabular}{lccc}
\hline Index & True value & Mean & STD \\
\hline$\hat{\rho}_{1}$ & 0.8000 & 0.7999 & 0.0020 \\
$\widehat{\rho}_{2}$ & 1.2500 & 1.2502 & 0.0045 \\
$\hat{\rho}_{3}$ & 0.7500 & 0.7503 & 0.0037 \\
\hline
\end{tabular}

(b) Phase estimation results of $\gamma_{t}$ (radian)

\begin{tabular}{lccc}
\hline Index & True value & Mean & STD \\
\hline$\widehat{\phi}_{1}$ & 0.6283 & 0.6285 & 0.0030 \\
$\widehat{\phi}_{2}$ & -1.0472 & -1.0468 & 0.0042 \\
$\widehat{\phi}_{3}$ & 0.7854 & -0.7859 & 0.0057 \\
\hline
\end{tabular}

(c) Gain estimation results of $\gamma_{r}$

\begin{tabular}{lccc}
\hline Index & True value & Mean & STD \\
\hline$\widehat{\alpha}_{1}$ & 1.3712 & 1.3722 & 0.0053 \\
$\widehat{\alpha}_{2}$ & 1.1199 & 1.1212 & 0.0068 \\
$\widehat{\alpha}_{3}$ & 1.0000 & 1.0016 & 0.0081 \\
$\widehat{\alpha}_{4}$ & 0.9730 & 0.9744 & 0.0086 \\
$\widehat{\alpha}_{5}$ & 1.0000 & 1.0018 & 0.0119 \\
$\widehat{\alpha}_{6}$ & 0.9600 & 0.9622 & 0.0132 \\
\hline
\end{tabular}

(d) Phase estimation results of $\gamma_{r}$ (radian)

\begin{tabular}{lccc}
\hline Index & True value & Mean & STD \\
\hline$\widehat{\beta}_{1}$ & -0.2675 & -0.2677 & 0.0037 \\
$\widehat{\beta}_{2}$ & 0.0676 & 0.0682 & 0.0064 \\
$\widehat{\beta}_{3}$ & 0 & 0.0000 & 0.0084 \\
$\widehat{\beta}_{4}$ & -0.2509 & -0.2500 & 0.0094 \\
$\widehat{\beta}_{5}$ & 0 & -0.0011 & 0.0123 \\
$\widehat{\beta}_{6}$ & 0.0381 & 0.0392 & 0.0147 \\
\hline
\end{tabular}

To evaluate the performance of the proposed method, we utilize the root mean square error (RMSE) result in the following simulations. In this paper, the RMSE is calculated from the following formula

$$
\mathrm{RMSE}=\sqrt{\frac{1}{K P} \sum_{p=1}^{L} \sum_{k=1}^{K}\left[\left(\widehat{\theta}_{k, p}-\theta_{k}\right)^{2}+\left(\hat{\phi}_{k, p}-\phi_{k}\right)^{2}\right]},
$$

where $P$ is the number of Monte Carlo trials and $\widehat{\theta}_{k, p}$ and $\widehat{\phi}_{k, p}$ are the estimations of $\theta_{k}$ and $\phi_{k}$ of the $p$ th trial, respectively. In the following experiments, we use $P=200$ independent trails to calculate the RMSE of the angle estimation results.

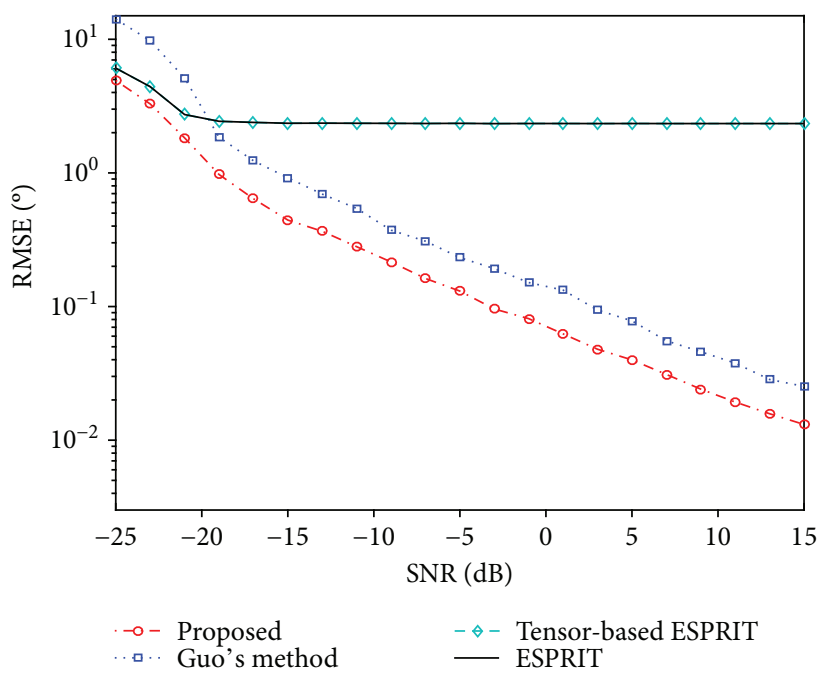

Figure 2: RMSE versus SNR.

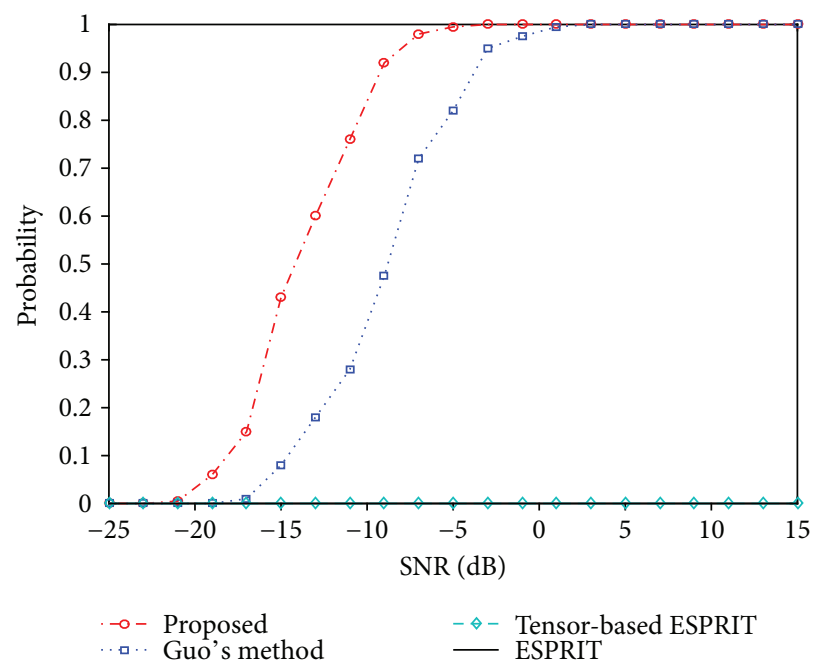

Figure 3: Probability of successful detection.

In Figure 2, the RMSEs of the conventional ESPRIT algorithm, tensor-based ESPRIT algorithm, method in [16] (denoted as Guo's method), and the proposed algorithm are compared with different SNRs. Due to the partially calibrated arrays, the conventional ESPRIT algorithm and the tensorbased ESPRIT algorithm fail to achieve accurate angle estimations. The Guo's method [16] is robust under the partially calibrated array case, however, it has a worse RMSE performance. On the other hand, the proposed method achieves the best estimation among the existing algorithms especially for the case of low SNR.

The probability of successful detection is provided in Figure 3. The successful detection means that the absolute error of all the DOA and DOD estimations is within $0.5^{\circ}$. As mentioned above, both the conventional ESPRIT and the tensor-based ESPRIT algorithms exhibit a 100\% failed detection due to the partially calibrated arrays used in this simulation. However, the proposed method achieves a higher probability of successful detection especially for 


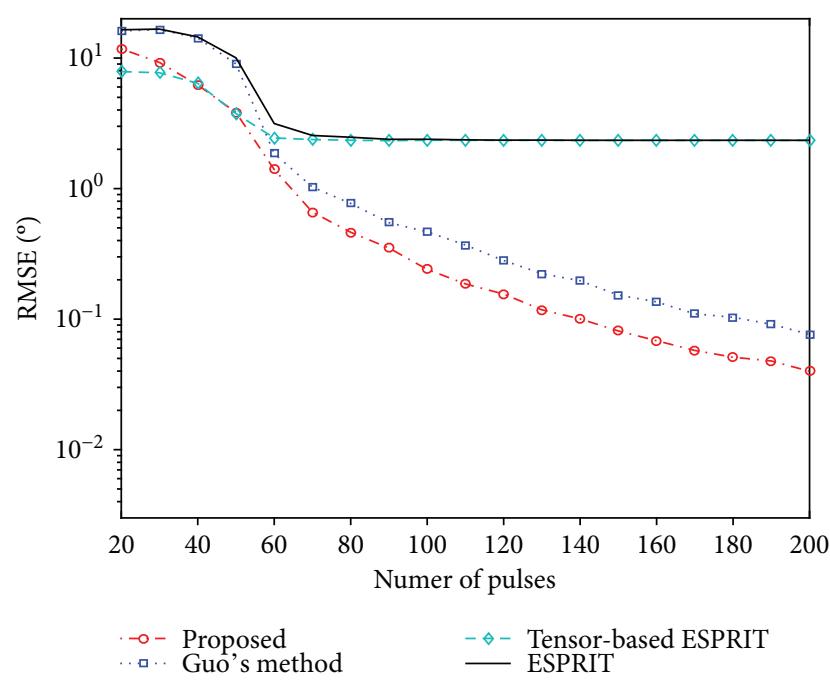

FIgURE 4: RMSE versus pulses.

SNR greater than $-20 \mathrm{~dB}$. This simulation result verifies the improved performance of the proposed algorithm over existing algorithms.

In Figure 4, we evaluate the performance of the algorithms under consideration for the case of varying number of the pulses. It is observed that the existing algorithms experience a heavily degraded estimation performance when the number of pulses is smaller than 60. In addition, both the proposed algorithm and Guo's method show improvement with the increased number of pulses. However, the proposed algorithm converges fast under low SNR and it always outperforms the other algorithms under consideration.

\section{Conclusion}

In this paper, we proposed a novel joint DOD-DOA estimation algorithm based on tensor subspace approach for the case of partially calibrated bistatic MIMO radar. We first develop a third-order measurement tensor of the received signal. The HOSVD is then exploited to evaluate the signal subspace of the tensor-based received signal matrix. A close-form expression for estimating the gain-phase uncertainties of the transmit and receive arrays is derived, which enables us to estimate the joint DOA-DOA effectively. Compared with the existing algorithms, the proposed algorithm achieves a significant improvement in estimation performance. Finally, the simulation results demonstrate the effectiveness of the proposed algorithm.

\section{Conflicts of Interest}

The authors declare that they have no conflicts of interest.

\section{Acknowledgments}

The work of Ping Huang is supported by the National Natural Science Foundation of China (61403091), the Heilongjiang Province Natural Science Foundation (QC2015049), and the launch of the postdoctoral research in Heilongjiang Province (LBH-Q16065).

\section{References}

[1] R. Schmidt, "Multiple emitter location and signal parameter estimation," IEEE Transactions on Antennas and Propagation, vol. 34, no. 3, pp. 276-280, 1986.

[2] Z. Shi, C. Zhou, Y. Gu, N. A. Goodman, and F. Qu, "Source estimation using coprime array: a sparse reconstruction perspective," IEEE Sensors Journal, vol. 17, no. 3, pp. 755-765, 2017.

[3] Y. Gu, N. A. Goodman, S. Hong, and Y. Li, "Robust adaptive beamforming based on interference covariance matrix sparse reconstruction," Signal Process, vol. 96, pp. 375-381, 2014.

[4] Y. Gu and A. Leshem, "Robust adaptive beamforming based on interference covariance matrix reconstruction and steering vector estimation," IEEE Transactions on Signal Processing, vol. 60, no. 7, pp. 3881-3885, 2012.

[5] H. Krim and M. Viberg, "Two decades of array signal processing research: the parametric approach," IEEE Signal Processing Magazine, vol. 13, no. 4, pp. 67-94, 1996.

[6] E. Fishler, A. Haimovich, R. S. Blum, L. J. Cimini, D. Chizhik, and R. A. Valenzuela, "MIMO radar: an idea whose time has come," in Proceedings of the 2004 IEEE Radar Conference (IEEE Cat. No.04CH37509), pp. 71-78, Philadelphia, PA, USA, 2004.

[7] J. Li and P. Stoica, "MIMO radar with colocated antennas," IEEE Signal Processing Magazine, vol. 24, no. 5, pp. 106-114, 2007.

[8] C. Duofang, C. Baixiao, and Q. Guodong, "Angle estimation using ESPRIT in MIMO radar," Electronics Letters, vol. 44, no. 12 , p. $770,2008$.

[9] C. Jinli, G. Hong, and S. Weimin, "Angle estimation using ESPRIT without pairing in MIMO radar," Electronics Letters, vol. 44, no. 24, p. 1422, 2008.

[10] X. Zhang, L. Xu, L. Xu, and D. Xu, "Direction of departure (DOD) and direction of arrival (DOA) estimation in MIMO radar with reduced-dimension MUSIC," IEEE Communications Letters, vol. 14, no. 12, pp. 1161-1163, 2010.

[11] M. L. Bencheikh, Y. Wang, and H. He, "Polynomial root finding technique for joint DOA DOD estimation in bistatic MIMO radar," Signal Processing, vol. 90, no. 9, pp. 27232730, 2010.

[12] R. Xie, Z. Liu, and J. Wu, "Direction finding with automatic pairing for bistatic MIMO radar," Signal Processing, vol. 92, no. 1, pp. 198-203, 2012.

[13] B. Friedlander and A. Weiss, "Eigenstructure methods for direction finding with sensor gain and phase uncertainties," in ICASSP-88., International Conference on Acoustics, Speech, and Signal Processing, pp. 2681-2684, New York, NY, USA, April 1988.

[14] A. Liu, G. Liao, C. Zeng, Z. Yang, and Q. Xu, "An eigenstructure method for estimating DOA and sensor gain-phase errors," IEEE Transactions on Signal Processing, vol. 59, no. 12, pp. 5944-5956, 2011.

[15] B. Liao and S. C. Chan, "Direction finding with partly calibrated uniform linear arrays," IEEE Transactions on Antennas and Propagation, vol. 60, no. 2, pp. 922-929, 2012. 
[16] Y. D. Guo, Y. S. Zhang, and N. N. Tong, "ESPRIT-like angle estimation for bistatic MIMO radar with gain and phase uncertainties," Electronics Letters, vol. 47, no. 17, p. 996, 2011.

[17] B. Wang, W. Wang, Y. Gu, and S. Lei, "Underdetermined DOA estimation of quasi-stationary signals using a partlycalibrated array," Sensors, vol. 17, no. 4, p. 702, 2017.

[18] B. Wang, Y. D. Zhang, and W. Wang, "Robust DOA estimation in the presence of miscalibrated sensors," IEEE Signal Processing Letters, vol. 24, no. 7, pp. 1073-1077, 2017.

[19] B. Wang, S. Qin, Y. D. Zhang, and M. G. Amin, "Robust DOA estimation in the presence of mis-calibrated sensors," in 2017 IEEE International Conference on Acoustics, Speech and Signal Processing (ICASSP), pp. 3076-3080, New Orleans, LA, USA, March 2017.

[20] D. Nion and N. D. Sidiropoulos, "Tensor algebra and multidimensional harmonic retrieval in signal processing for MIMO radar," IEEE Transactions on Signal Processing, vol. 58, no. 11, pp. 5693-5705, 2010.

[21] T. G. Kolda and B. W. Bader, "Tensor decompositions and applications," SIAM Review, vol. 51, no. 3, pp. 455-500, 2009.

[22] M. Skolnik, Introduction to Radar Systems, McGraw-Hill, New York, NY, USA, 3rd edition, 2002. 


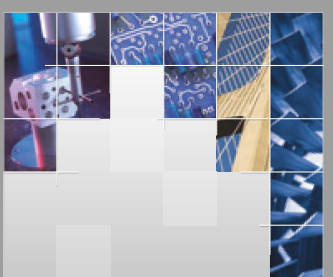

\section{Enfincering}
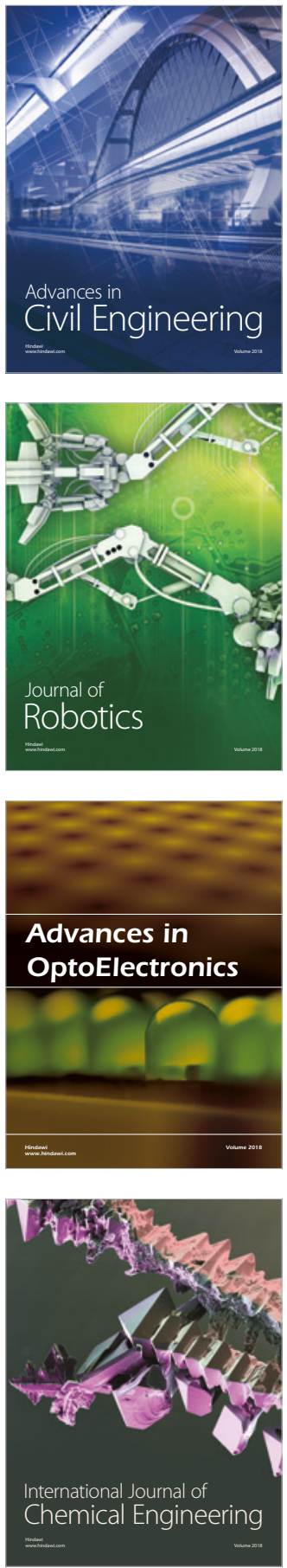

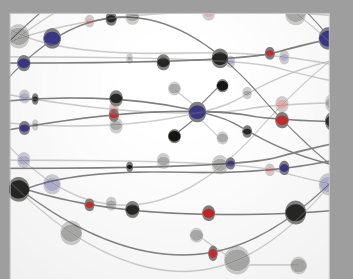

\section{Rotating \\ Machinery}

The Scientific World Journal

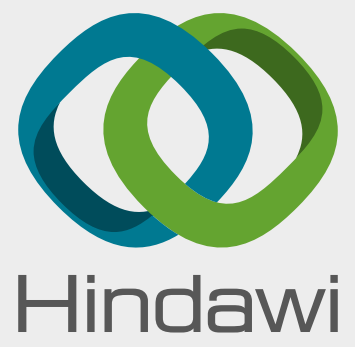

Submit your manuscripts at

www.hindawi.com
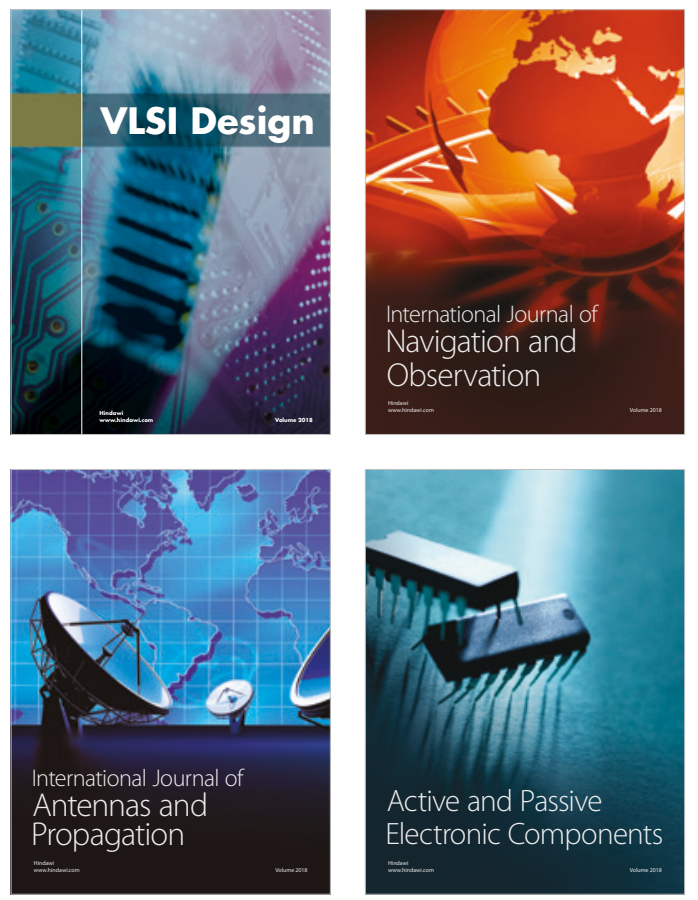
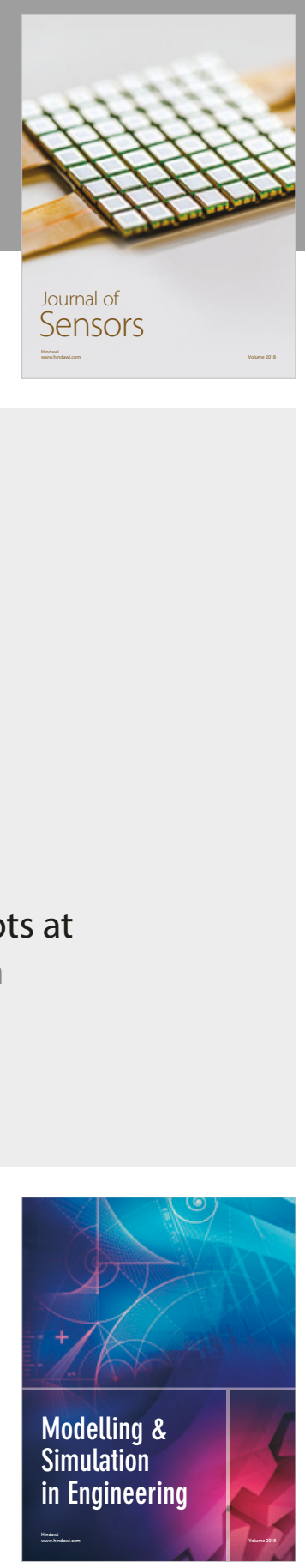

\section{Advances \\ Multimedia}
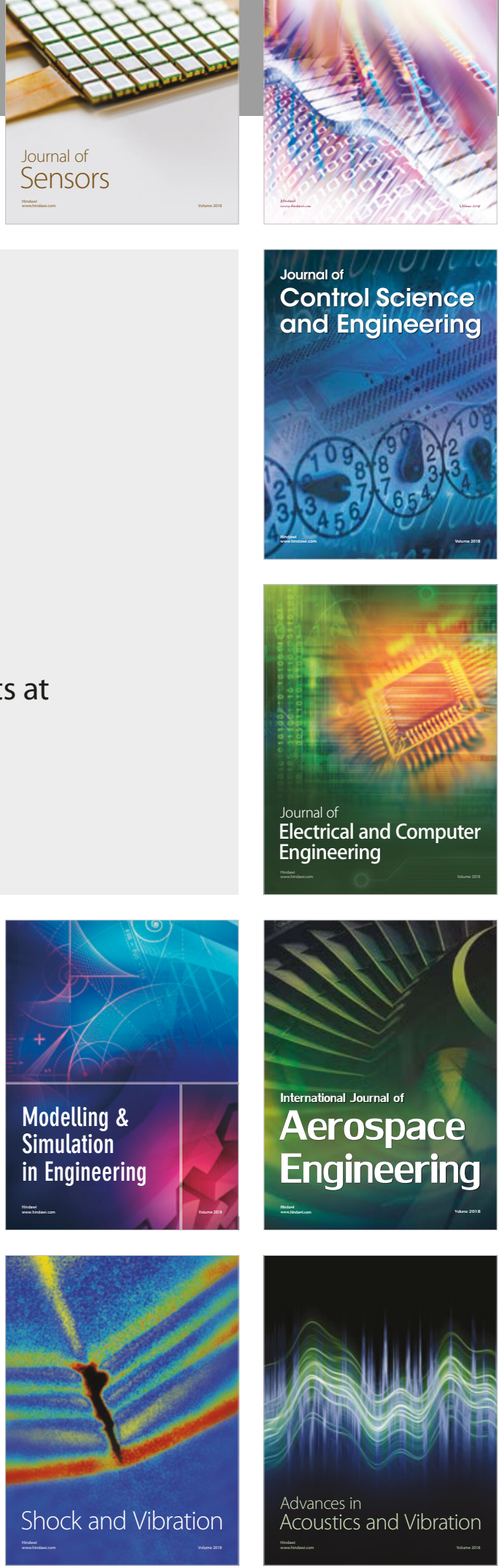